\title{
Comparaison de différents modèles d'ajustement des courbes d'excrétion fécale des marqueurs du transit
}

\author{
T Najar ${ }^{1}$, D Sauvant ${ }^{1}$, C Poncet ${ }^{2}$, S Giger-Reverdin ${ }^{1}$ \\ 1 Station de Nutrition et d'Alimentation (INRA) de I'INA-PG, 16, rue C-Bernard, \\ 75231 Paris Cedex 05; 2 Station de Nutrition des Herbivores, INRA, Theix, 63122 Ceyrat, France
}

\begin{abstract}
Summary - Seventy nine fecal excretion curves of rare earth were adjusted with 3 published models (Grovum and Williams, 1973; Dhanoa et al, 1985; Siddons et al, 1983). The models provided statistically similar estimations of particulate turnover in the first compartment and mean retention time. However, some important individual differences between parameter values could be observed from one model to another.
\end{abstract}

Introduction - Différents modèles ont été proposés pour ajuster les courbes d'excrétion fécale des marqueurs du transit digestif et en déduire des estimations des temps de séjour moyen (TSM) des aliments dans le tube digestif et dans ses compartiments. Les résultats obtenus par 3 de ces modèles ont été comparés à ceux calculés par la méthode de référence des moments.

Matériel et Méthodes - Un ensemble de 79 courbes d'excrétion fécale ont été obtenues sur des chèvres produisant de 0 à $5,8 \mathrm{~kg}$ de lait $j$ et ingérant des rations dont les 2 ou 3 constituants (foin de luzerne, pulpe de betteraves ensilée, aliment concentré) ont été marqués séparément avec des terres rares (respectivement Yb, Dy, Eu), selon la technique de Poncet et al (1987). Les cinétiques d'évolution des concentrations fécales des marqueurs ont été ajustées par le modèle à 2 compartiments (GW) de Grovum et Williams (1973), par le modèle multicompartimental (MD) de Dhanoa et al (1985) et par sa transformation logarithmique (MS) proposée par Siddons et al (1983) pour permettre l'estimation des paramètres par régression linéaire. La variante du modèle GW à 3 compartiments a également été étudiée, elle n'est pas prise en compte dans la comparaison car ses paramètres supplémentaires ne lui confèrent pas d'avantage. Le TSM a également été calculé par la méthode des moments $\left(T S M_{M}\right)$. $Y_{t}$ représentant la concentration fécale du marqueur à un instant $t$, les formes analytiques des modèles sont:

$$
\begin{aligned}
& \text { GW : } Y_{1}=A_{1} \cdot e^{-K_{1}(t-T T)}-A_{2} \cdot e^{-K_{2}(t-T)} \\
& \text { MS : } \operatorname{Ln} Y_{t}: \operatorname{Ln}\left(A_{1}-A_{2}\right) \cdot e^{-K_{2}(t-T)} \cdot K_{1} t \\
& \text { MD : } Y_{t}=A_{1} \cdot e^{-K_{1} t} \cdot e^{-A_{2} \cdot e^{-K_{2} t}}
\end{aligned}
$$

Les comparaisons portent sur les TSM, et les taux de renouvellement $K_{1}$ et $K_{2}$. Le temps de première apparition du marqueur $(T T)$ correspond à la valeur observée qui est donc la même pour les 3 modèles.

Résultats et Discussion - Le tableau I rapporte les valeurs moyennes et les écarts types des paramètres des $3 \mathrm{mo}$ dèles, la moyenne de $T T$ est de $19,4 \mathrm{~h}$ et son écart type (ET) de $3,3 \mathrm{~h}$. Le $T S M_{M}$ est de $49,0(E T=5,0 \mathrm{~h})$, les valeurs de $T S M$ obtenues par les 3 modèles ne sont pas significativement différentes et lui sont positivement liées : $T S M_{\mathrm{GW}}=0,85 T S M_{\mathrm{M}}+$ $7,76(R=0,76, E T R=5,1 \mathrm{~h}) ; T S M_{M S}=$ 
Tableau I. Caractéristiques des paramètres obtenus avec les différents modèles a.

\begin{tabular}{|c|c|c|c|c|}
\hline Modèle & $\begin{array}{c}\mathrm{K} \\
\left(h^{-1}\right)\end{array}$ & $\begin{array}{c}\mathrm{K}_{2} \\
\left(t^{-1}\right)\end{array}$ & $\begin{array}{c}\text { TSM } \\
\text { (h) }\end{array}$ & $\begin{array}{c}\text { ETR } \\
(\mu g / g M S\end{array}$ \\
\hline GW & $\begin{array}{c}0,056 \\
(0,013)\end{array}$ & $\begin{array}{c}0,133 \\
(0,086)\end{array}$ & $\begin{array}{c}49,71 \\
(6,88)\end{array}$ & $\begin{array}{c}1,33 \\
\quad(1,28)\end{array}$ \\
\hline MS & $\begin{array}{c}0,049 \\
(0,0011)\end{array}$ & $\begin{array}{c}0,211 \\
(0,124)\end{array}$ & $\begin{array}{c}47,40 \\
(5,30)\end{array}$ & $\begin{array}{c}1,23 \\
(1,13)\end{array}$ \\
\hline MD & $\begin{array}{c}0,049 \\
(0,0013)\end{array}$ & $\begin{array}{c}0,205 \\
(0,143)\end{array}$ & $\begin{array}{c}47,64 \\
(5,32)\end{array}$ & $\begin{array}{c}0,91 \\
(0,59)\end{array}$ \\
\hline
\end{tabular}

a Ecarts type de la série des 79 mesures entre parenthèses.

$0,86 T_{S M}+6,68(R=0,78, E T R=3,4$ h); $T S M_{M D}=0,77 T S M_{M}+11,45(R=$ $0,70, E T R=5,7 \mathrm{~h}$ ). Dans ces relations les coefficients de pente ne sont pas significativement différents de 1 . Les valeurs des $E T$ résiduels (ETR) sont relativement élevées en particulier pour les modèles GW et MD; ce fait révèle des possibilités de différences importantes entre les estimations des TSM.

Les taux de renouvellement $K_{1}$ dans le compartiment 1 des modèles ne diffèrent pas et sont positivement corrélés $(P<$ $0,01): R(\mathrm{GW}, \mathrm{MS})=0,62 ; R(\mathrm{GW}, \mathrm{MD})=$ 0,$77 ; A(M S, M D)=0,75$; il en est de même des taux de renouvellement $K_{2}$ du $2^{\theta}$ compartiment : $A$ (GW, MS) : 0,62; $R$ $(G W, M D)=0,41 ; R(M S, M D)=0,76$. Par contre, les estimations de ces coefficients $K_{2}$ sont largement différentes entre le modèle GW et les modèles MS et MD. En fait, ce coefficient $K_{2}$ traduit la phase croissante de la courbe d'excrétion et n'est pas formalisé de la même manière entre le modèle GW, d'une part, et MS ou MD, d'autre part. Les valeurs de ces différents coefficients de corrélation révèlent également certains écarts importants entre les estimations de $K_{1}$ et $K_{2}$ selon le modèle considéré. Le modèle MD ajuste en moyenne significativement mieux les courbes d'excrétion des marqueurs que le modèle MS et surtout GW (tableau I) alors que Lallès et al (1989) n'avaient pas observé de différence sur des veaux entre les modèles GW et MD. Les courbes de concentration fécale du marqueur du foin sont, par rapport à celles de la pulpe et du concentré, moins bien ajustées par les modèles MD $(E T R=0,99$ vs 0,84$)$, MS ( $E T R=1,33$ vs $1,16)$ et surtout $\mathrm{GW}(E T R=1,58$ vs 1,17$)$.

En conclusion, les différents modèles aboutissent à des estimations moyennes identiques des temps de séjour moyens des particules dans le tube digestif et des taux de renouvellement dans le premier compartiment qui est souvent identifié au réticulo-rumen. Cependant, des différences individuelles non négligeables de valeur d'estimation de ces paramètres peuvent être observées selon la méthode utilisée.

Dhanoa M, Siddons RC, France J, Gale D (1985) Br J Nutr 53, 663-671

Grovum WL, Williams VJ (1973) Br J Nutr 30, 313-329

Lallès JP, Delval E, Poncet C (1990) Reprod Nutr Dev suppl 2, 223s-224s

Poncet C, Gomez L, Michalet-Doreau B, Geay Y (1987) Reprod Nutr Dev 27, 219-220

Siddons RC, Beever DE, Paradine J (1983) Grass Res Inst Annu Repp 15 